\title{
Linked color imaging improves the visibility of colorectal polyps: a video study $\square$
}

\section{(ㄷ)(1) $\odot$}

\section{Authors}

Naohisa Yoshida', Yuji Naito', Takaaki Murakami ${ }^{1}$, Ryohei Hirose ${ }^{1}$, Kiyoshi Ogiso ${ }^{1}$, Yutaka Inada ${ }^{1}$, Osamu Dohi ${ }^{1}$, Kazuhiro Kamada', Kazuhiko Uchiyama ${ }^{1}$, Osamu Handa ${ }^{1}$, Hideyuki Konishi', Kewin Tien Ho Siah², Nobuaki Yagi ${ }^{3}$, Yasuko Fujita ${ }^{4}$, Mitsuo Kishimoto ${ }^{5}$, Akio Yanagisawa ${ }^{5}$, Yoshito Itoh ${ }^{1}$

\section{Institutions}

1 Department of Molecular Gastroenterology and Hepatology, Kyoto Prefectural University of Medicine, Graduate School of Medical Science, Kyoto, Japan

2 Division of Gastroenterology \& Hepatology, University Medicine Cluster, National University Hospital, Singapore

3 Department of Gastroenterology, Murakami Memorial Hospital, Gifu, Japan

4 Department of Pathology and Cell Regulation, Kyoto Prefectural University of Medicine, Graduate School of Medical Science, Kyoto, Japan

5 Department of Surgical Pathology, Kyoto Prefectural University of Medicine, Graduate School of Medical Science, Kyoto, Japan

submitted 2.10.2016

accepted after revision 13.2.2017

\section{Bibliography}

DOI https://doi.org/10.1055/s-0043-105495 |

Endoscopy International Open 2017; 05: E518-E525

(c) Georg Thieme Verlag KG Stuttgart · New York ISSN 2364-3722

Corresponding author Naohisa Yoshida, MD PhD, Department of Molecular Gastroenterology and Hepatology, Kyoto Prefectural University of Medicine, Graduate School of Medical Science, 465 Kajii-cho, Kawaramachi-Hirokoji, Kamigyo-ku, Kyoto 602-8566, Japan

Fax: +81-75-2510710

naohisa@koto.kpu-m.ac.jp

\section{ABSTRACT}

Background/study aim Linked color imaging (LCI) by a laser endoscope (Fujifilm Co, Tokyo, Japan) is a novel narrow band light observation. In this study, we aimed to investigate whether $\mathrm{LCl}$ could improve the visibility of colorectal polyps using endoscopic videos.

Patients and methods We prospectively recorded videos of consecutive polyps $2-20 \mathrm{~mm}$ in size diagnosed as neoplastic polyps. Three videos, white light (WL), blue laser imaging (BLI)-bright, and $\mathrm{LCl}$, were recorded for each polyp by one expert. After excluding inappropriate videos, all videos were evaluated in random order by two experts and two non-experts according to a published polyp visibility score from four (excellent visibility) to one (poor visibility). Additionally, the relationship between polyp visibility scores in $\mathrm{LCl}$ and various clinical characteristics including location, size, histology, morphology, and preparation were analyzed compared to WL and BLI-bright.

Results We analyzed 101 colorectal polyps (94 neoplastic) in 66 patients (303 videos). The mean polyp size was $9.0 \pm$ $8.1 \mathrm{~mm}$ and 54 polyps were non-polypoid. The mean polyp visibility scores for $\mathrm{LCl}(2.86 \pm 1.08)$ were significantly higher than for $W L$ and BLI-bright $(2.53 \pm 1.15, P<0.001 ; 2.73 \pm$ $1.47, P<0.041$ ). The ratio of poor visibility (score 1 and 2 ) was significantly lower in $\mathrm{LCl}$ for experts and non-experts (35.6\%, 33.6\%) compared with WL $(49.6 \%, P=0.015$, $50.5 \%, P=0.046)$. The polyp visibility scores for $\mathrm{LCl}$ were significantly higher than those for WL for all of the factors. With respect to the comparison between BLI-bright and WL, the polyp visibility scores for BLI-bright were not higher than WL for right-sided location, $<10 \mathrm{~mm}$ size, sessile serrated adenoma and polyp histology, and poor preparation. For those characteristics, LCI improved the lesions with right-sided location, SSA/P histology, and poor preparation significantly better than $\mathrm{BLI}$.

Conclusions LCl improved polyp visibility compared to WL for both expert and non-expert endoscopists. It is useful for improving polyp visibility in any location, any size, any morphology, any histology, and any preparation level. Study registration: UMIN000013770 


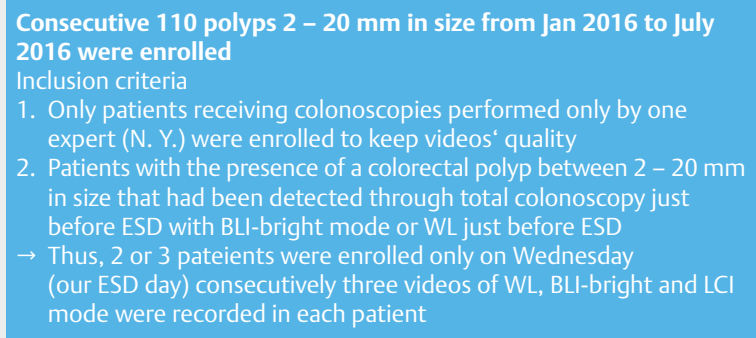

Finally, 101 polyps in 66 patients were analyzed (303 videos: 101 WI, 101 LCI, 101 BLI-bright videos)

$\downarrow$

All 303 videos were viewed in a randomized order by the 4 endoscopists

For example, Video number 1 . Polyp A's $s$ Cl video $\rightarrow$ Video number 2 . Polyp B's WL video $\rightarrow$ Video number 3. Polyp C's BLI-bright video $\rightarrow$...

Fig. 1 A flow diagram of this study.

\section{Introduction}

The number of colorectal cancer deaths is increasing in the West and in Asian countries. The adenoma-carcinoma sequence is thought to be one of the main pathways for the development of colorectal cancers [1]. Therefore, adenomas are resected using endoscopic treatments such as polypectomy, endoscopic mucosal resection (EMR), and endoscopic submucosal dissection (ESD) [2-4]. Colonoscopy is the most effective tool for detecting colorectal adenomas. However, the polyp miss rate under white light (WL) observation was reported to be $20-25 \%$ [5]. Therefore, narrow-band imaging (NBI; Olympus Medical Co., Tokyo, Japan), flexible spectral imaging color enhancement (FICE; Fujifilm Co., Tokyo, Japan), and chromoendoscopy were investigated to determine whether they could improve the polyp detection rate; however, most of them failed [6-11].

A LASER endoscope system was developed in 2012. There were two modes of narrow-band imaging observation in that system: blue laser imaging (BLI; Fujifilm Co., Tokyo, Japan) mode and BLI-bright mode [12-15]. Previously, we have reported that BLI-bright improved polyp visibility compared with WL using recorded polyp videos [16]. To make a polyp more visible is considered to be one of the most important factors related to efficient polyp detection. Recently, new narrowband light observation, named linked color imaging (LCI), has been developed for diagnosing chronic gastritis in the LASER endoscope [17]. It is brighter than BLI-bright and may also improve colorectal polyp detection.

In the present study, we aimed to investigate whether $\mathrm{LCl}$ could improve the visibility of colorectal polyps compared to WL and BLI-bright using endoscopic videos.

\section{Patients and methods}

This was a prospective study and was conducted at the Department of Molecular Gastroenterology and Hepatology, Kyoto Prefectural University of Medicine. We examined consecutive colorectal polyps diagnosed as neoplastic polyps and captured videos of the polyps using three modes ( $\mathrm{LCl}$, BLI-bright, and $\mathrm{WL}$ ) with the LASER endoscope system (LL-4450 light source, and VP-4450HD video processor, Fujifilm Co., Tokyo, Japan) from January 2016 to July 2016. The inclusion criteria were as follows: (1) patients receiving colonoscopies performed only by one expert (N.Y.) were enrolled to retain video quality; (2) patients with colorectal polyps diagnosed as neoplastic polyps between $2 \mathrm{~mm}$ and $20 \mathrm{~mm}$ in size that had been detected through colonoscopy just before ESD with BLI-bright mode or WL ( $\triangleright$ Fig. 1).

Diagnosis of neoplastic polyps was performed with BLI magnification according to previous reports $[12,13,18]$. Thus, two or three patients were enrolled consecutively only on Wednesdays (our ESD day). In each patient, the number of recorded polyps was limited to a maximum of three on account of patient fatigue during the recording of videos. Three videos in WL, BLIbright, and $\mathrm{LCl}$ mode were recorded for each polyp ( $\triangleright$ Fig. 1). Each video included a range of $3-5 \mathrm{~cm}$ proximal to the polyp to $3-5 \mathrm{~cm}$ distal to the polyp (a 5- to 10 -second clip). Three videos were recorded for a polyp under the same conditions if possible about the presence of fluid, the amount of insufflation, and the withdrawal speed. Polyp visibility was evaluated using a published polyp visibility score [16] ranging from Score 4 to Score 1 . Score 4 indicates excellent visibility; it is easy to detect a polyp. Score 3 indicates good visibility. If an endoscopist looks in the same direction as a polyp in the monitor, it is easy to detect the polyp. Score 2 indicates fair visibility. It is hard to detect the polyp without careful observation. Score 1

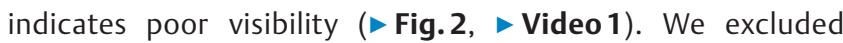
polyps with noticeable variations of recorded conditions (air, speed, fluid, etc.). Additionally, videos were excluded when more than two polyps were present in the same video. Recurrent lesions after a previous EMR or T2 - T4 colorectal cancers were also excluded.

The polyp locations were divided into three parts: the rightsided colon (from the cecum to the transverse colon), the leftsided colon (from the descending colon to the sigmoid colon), and the rectum. With regard to morphology, polyps were divided into polypoid and non-polypoid according to the Paris classification [19]. The size of a polyp was defined by its maximum diameter and was calculated in accordance with the size of the snares.

All of the sets of videos taken during the study period were collected. The evaluation was performed by four endoscopists who had not viewed any of these videos before this study. Of the four endoscopists, two were classified as non-experts (had performed $<5000$ colonoscopies and $10-30$ withdrawing colonoscopies with BLI-bright and $\mathrm{LCl}$ ) and two were classified as experts (had performed $\geq 5000$ colonoscopies and 300 withdrawing colonoscopies with BLI-bright and $\mathrm{LCI}$ ) according to a previous report [9]. All of the videos were viewed in a random- 


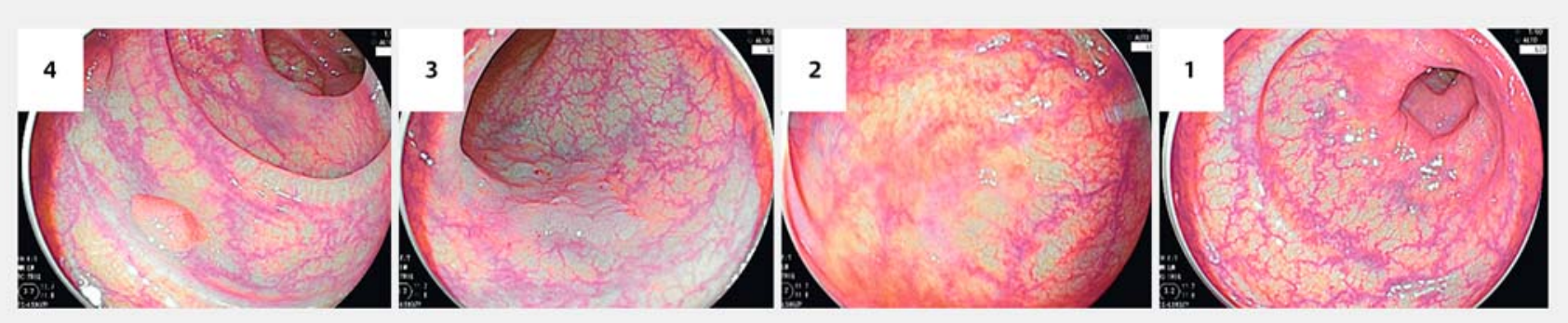

Fig. 2 Examples of polyp visibility score. Score 4, excellent visibility; score 3, good visibility; score 2, fair visibility; score 1, poor visibility.

ized order. For example, Video number 1, a LCI video of polyp A, was followed by Video number 2, which was a WL video of polyp B. Each endoscopist assigned a polyp visibility score to each polyp. Scores for each mode (WL, BLI-bright and $\mathrm{LCl}$ ) were compared and the ratios of polyps with poor visibility (scores 1 and 2) for experts and non-experts in each mode were examined. In addition, the mean polyp visibility scores of $\mathrm{WL}, \mathrm{LCl}$ and BLIbright mode in terms of various clinical characteristics including location (right-sided or not right-sided), size ( $\geq 10 \mathrm{~mm}$ or $<10 \mathrm{~mm}$ ), histology (adenoma + intramucosal cancer or sessile serrated adenoma and polyp (SSA/P)), morphology (polypoid or non-polypoid), and preparation (good or poor) were analyzed. With respect to preparation level, good preparation was defined as local Boston bowel preparation score (BBPS) 2 or 3 , and poor preparation was defined as local BBPS 0 or 1 . Moreover, the inter-observer agreements of polyp visibility score for experts and non-experts in each mode were analyzed for objective evaluation of polyp visibility scores.

With regard to bowel preparation, patients followed a lowresidue diet and were given $10 \mathrm{~mL}$ sodium picosulfate 1 day before the examination. All patients also received $1.0 \mathrm{~L}$ of a highly concentrated polyethylene glycol solution with ascorbic acid (MoviPrep; Ajinomoto Pharma Co., Ltd, Tokyo, Japan) in the morning on the day of the examination according to our previous report [20].

All patients provided written informed consent to participate in this study. This study was conducted in accordance with the World Medical Association Helsinki Declaration. It was also approved by the institutional review board and the ethics committees of Kyoto Prefectural University of Medicine. In addition, this study was a subgroup analysis of a study registered in the University Hospital Medical Information Network Clinical Trials Registry (UMIN-CTR) as number UMIN000013770.

\section{Histological diagnosis}

The tumor specimens were obtained by polypectomy and EMR. Thereafter, they were fixed with $10 \%$ formalin and evaluated histologically. Histological diagnosis was performed by two clinical pathologists (M.K. and A. Y.) according to the World Health Organization classification [21]. Thus, polyps were divided into neoplastic or non-neoplastic categories based on the histological diagnosis. SSA/P were defined as neoplastic lesions in this study.

\section{LASER endoscope and $\mathrm{LCI}$ mode}

The LASER endoscope system used in this study has been described in previous reports $[12,13]$. In brief, this system uses a semiconductor laser as the light source and has a narrow-band light observation function called BLI and $\mathrm{LCI}$. It has two types of laser with $410 \mathrm{~nm}$ and $450 \mathrm{~nm}$ wavelengths. There are two modes for BLI, i.e. BLI mode and BLI-bright mode. BLI mode is useful for acquiring magnified mucosal surface vessels and structure. BLI-bright mode is brighter than BLI mode and it is expected to be useful in tumor detection. However, the weakness of $\mathrm{BLI}$ is that the residual liquid becomes reddish. $\mathrm{LCI}$ mode is a novel mode and is based on the image captured by light similar to BLI-bright mode; however, further post image processing is applied so that the strong red-tint color becomes more reddish and the pale red-tint color becomes paler. This difference between red color and pale color makes a lesion more noticeable and $\mathrm{LCl}$ is brighter than BLI-bright mode ( $>$ Fig.3). In addition, the residual liquid was not reddish in $\mathrm{LCI}$ mode compared to BLI-bright mode.

\section{Statistical assessment}

In a pilot study on the visibility of 33 colorectal polyps by a single expert endoscopist, $\mathrm{LCl}$ achieved superior polyp visibility scores compared with WL in 15 polyps (15/33; 45.4\%). Using a sign test, the $\alpha$ error was $5 \%$ and $\beta$ error was $20 \%$. Thus, the minimum sample size was calculated to be 25 . The pilot study

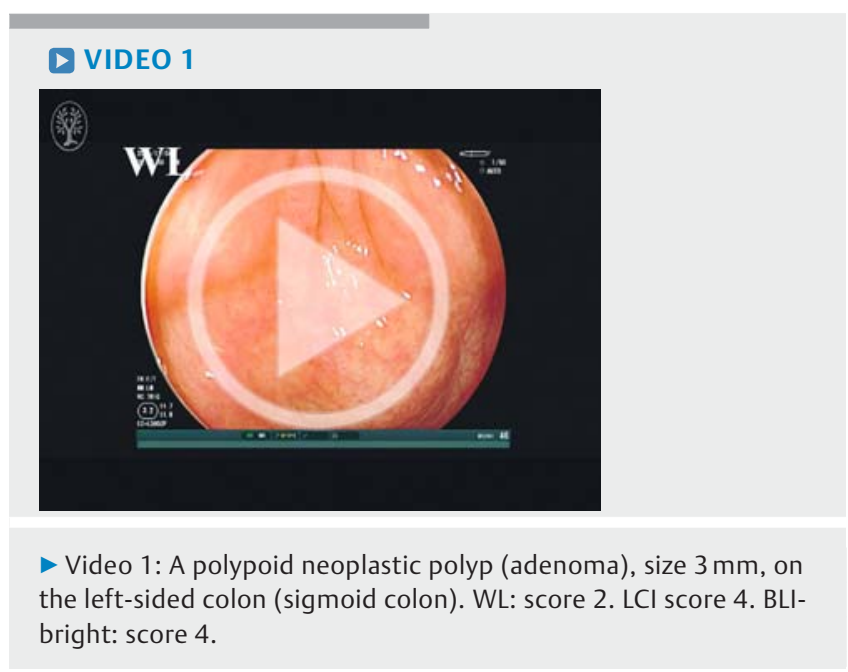



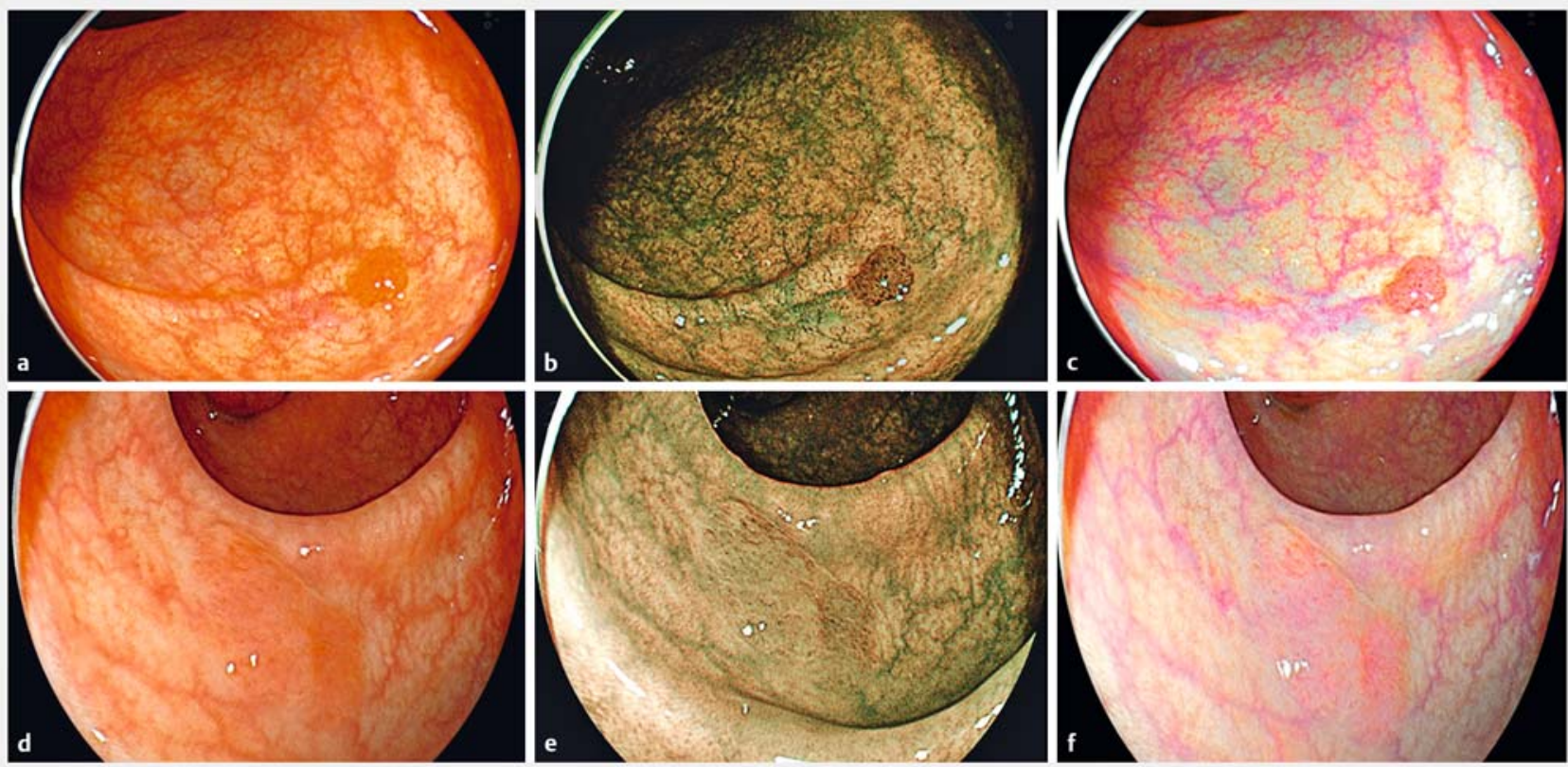

- Fig. 3 Case presentation. a A polypoid neoplastic polyp (adenoma), $3 \mathrm{~mm}$ on the left-sided colon (sigmoid colon). WL: score 2. b BLI-bright: score 4. c LCl score 4. d A non-polypoid polyp (SSA/P), $8 \mathrm{~mm}$ in size on the right-sided colon (transverse colon), WL: score 2. e BLI-bright: score 3. f LCl: score 3 .

was performed by one expert and subgroup analysis was designed for location, polyp size, etc. Thus, we decided to use a sample size of 100 . The Mann-Whitney $U$ test, Wilcoxon signed-rank test with Bonferroni correction, and the chisquared test (SPSS version 22.0 for Windows, IBM Japan, Ltd., Tokyo, Japan) were used in this study. Continuous variables such as patient age and tumor size were analyzed using the Mann-Whitney $U$ test. The four phases of the evaluation (polyp visibility scores $1-4$ ) were used as ordered-categorical variables, and comparisons between methods used the Wilcoxon signed-rank test with Bonferroni correction. Inter-observer agreement for polyp visibility scores was determined by the quadratic-weighted kappa coefficient of Cohen. A $P$ value $<$ 0.05 was considered to be statistically significant.

\section{Results}

In total, videos from 110 consecutive polyps (WL, LCl, and BLIbright) were recorded and nine polyps were excluded according to the decided criteria ( $\mathbf{F i g .} \mathbf{1}$ ). Finally, a total of 101 polyps (mean polyp size $9.0 \pm 8.1 \mathrm{~mm}$ ) in 66 patients (303 videos) were investigated in this study ( $\bullet$ Table 1 ). Forty-seven polyps ( $46.5 \%$ ) were polypoid and 55 polyps $(54.5 \%$ ) were in the right-sided colon. Ninety-four polyps (93.1\%) were neoplastic $(\triangleright$ Table 1$)$.

The mean polyp visibility scores of LCI mode $(2.86 \pm 1.08)$ were significantly higher than those of WL $(2.53 \pm 1.15, P<$ $0.001)$ and BLI-bright $(2.73 \pm 1.47, P=0.041)$ ( $\triangleright$ Table 2). For non-experts, the polyp visibility scores of $\mathrm{LCl}(2.83 \pm 1.07)$ were higher than WL $(2.51 \pm 1.14, P<0.001)$ and BLI-bright
Table 1 Clinical characteristics of 101 colorectal polyps.

\begin{tabular}{|c|c|}
\hline Number of polyps & 101 \\
\hline Number of patients & 66 \\
\hline Sex (male/female) & $45 / 21$ \\
\hline Age, mean \pm SD (range), years & $67.7 \pm 9.8(39-85)$ \\
\hline Polyp size, mean \pm SD (range), mm & $9.0 \pm 8.1(2-20)$ \\
\hline $\begin{array}{l}\text { Location, (right-sided: left-sided: } \\
\text { rectum), } \mathrm{n}(\%)\end{array}$ & $55: 29: 17(54.5: 28.7: 16.8)$ \\
\hline $\begin{array}{l}\text { Morphology (polypoid or } \\
\text { non-polypoid), } n \text { (\%) }\end{array}$ & $47: 54(46.5: 53.5)$ \\
\hline
\end{tabular}

Histopathological diagnosis

- Non-neoplastic/neoplastic, n (\%) 7 (6.9):94 (93.1)

\begin{tabular}{ll|l} 
- HP:SSA/P:Ad:Tis, $n$ & 7:20:51:23
\end{tabular}

Right-sided: from the cecum to the transverse colon; left-sided: from the descending colon to the sigmoid colon; SSA/P: sessile serrated adenoma/ polyp; HP: hyperplastic polyp; Ad: adenoma; Tis: intramucosal cancer.

$(2.70 \pm 1.12, P=0.047)$. On the other hand, for experts, the polyp visibility scores of $\mathrm{LCl}(2.83 \pm 1.07)$ were higher than WL $(2.58 \pm 1.19, P<0.001)$, but not significantly higher than BLIbright $(2.78 \pm 1.17, P=0.30)$ ( $\triangleright$ Table 2 ).

The ratios of poor visibility (score 1 or 2 ) of WL, BLI-bright, and $\mathrm{LCl}$ for experts and non-experts were analyzed ( $\triangleright$ Fig.4). For the experts, the mean ratio of poor visibility was significantly lower in $\mathrm{LCl}(16.8+18.8: 35.6 \%)$ than in WL mode $(24.8$ 
- Table 2 Mean polyp visibility scores of colorectal polyps in WL, LCI, and BLI-bright mode for all endoscopists, experts, and non-experts.

\begin{tabular}{|c|c|c|c|c|}
\hline & WL & LCI & BLI-bright & $P$ value \\
\hline All & $2.53 \pm 1.15$ & $2.86 \pm 1.08$ & $2.73 \pm 1.47$ & $\begin{array}{l}\text { WL vs. LCI } P<0.001 \\
\text { WL vs. BLI-bright } P=0.005 \\
\text { LCI vs. BLI-bright } P=0.041\end{array}$ \\
\hline Experts & $2.58 \pm 1.19$ & $2.87 \pm 1.12$ & $2.78 \pm 1.17$ & $\begin{array}{l}\text { WL vs. LCI } P<0.001 \\
\text { WL vs. BLI-bright } P=0.01 \\
\text { LCl vs. BLI-bright } P=0.30\end{array}$ \\
\hline Non-experts & $2.51 \pm 1.14$ & $2.83 \pm 1.07$ & $2.70 \pm 1.12$ & $\begin{array}{l}\text { WL vs. LCI } P<0.001 \\
\text { WL vs. BLI-bright } P=0.006 \\
\text { LCI vs. BLI-bright } P=0.047\end{array}$ \\
\hline
\end{tabular}

WL: white light; LCI: linked color imaging; BLI: blue laser imaging.

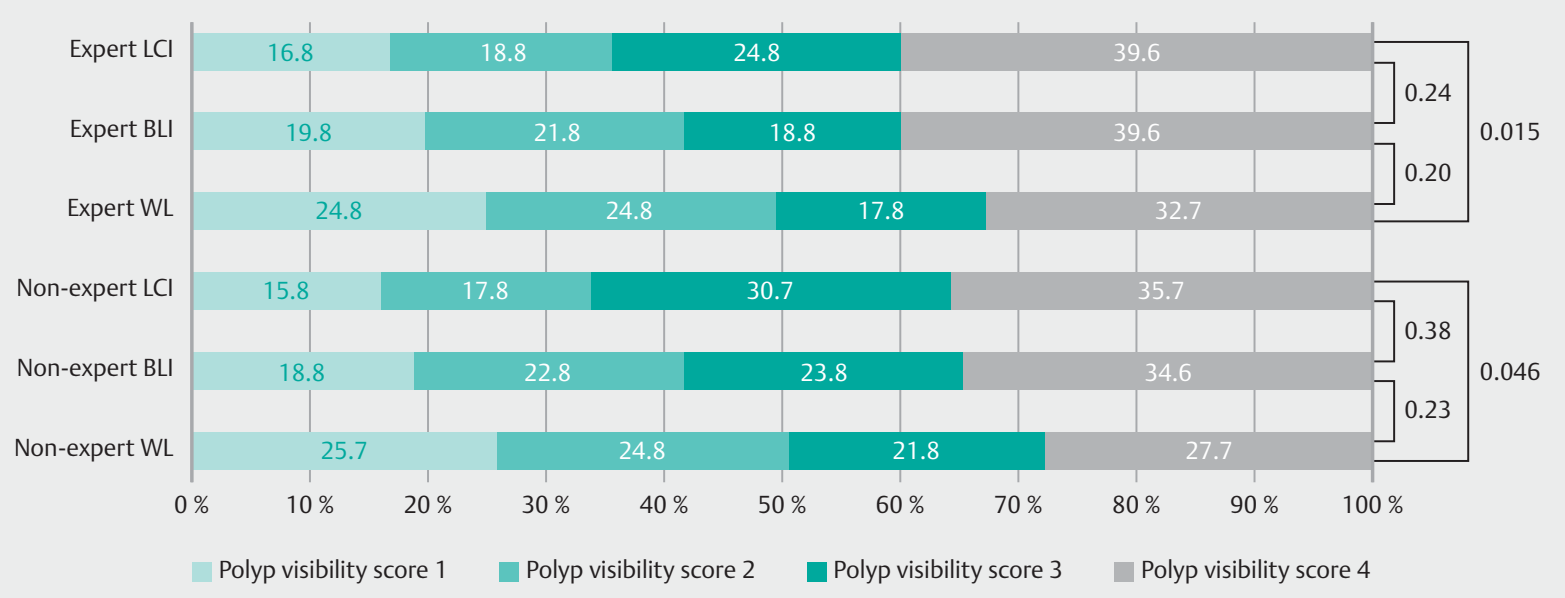

Fig. 4 Graphic for derivation of the ratios of poor polyp visibility scores (1 or 2) in LCI, BLI-bright, and WL modes.

+ 24.8: 49.6\%) $(P=0.015)$. For the non-experts, the mean ratio of poor visibility in $\mathrm{LCl}(15.8+17.8$ : $33.6 \%)$ was also significantly lower than that in WL $(25.7+24.8: 50.5 \%)(P=0.046)$.

The mean polyp visibility scores of $\mathrm{WL}, \mathrm{LCl}$ and BLI-bright mode in terms of various clinical characteristics are shown in - Table 3. The polyp visibility scores for LCI mode were significantly higher than those for WL for all of the factors. With respect to the comparison between BLI and WL, the polyp visibility scores of $\mathrm{BLI}$ were not higher than $\mathrm{WL}$ in right-sided location, $<10 \mathrm{~mm}$ size, SSA/P histology, and poor preparation. For those clinical characteristics, the visibility scores of lesions with rightsided location, SSA/P histology, and poor preparation were significantly higher with LCl than with BLI ( $\triangleright$ Fig.5).

With respect to the analysis of inter-observer agreement, the kappa values for the four endoscopists (expert A vs expert $B$, non-expert $A$ vs non-expert $B$ ) were calculated in $L C l, W L$, and BLI-bright mode. The kappa values for experts were 0.87 for $\mathrm{LCl}, 0.91$ for $\mathrm{WL}$, and 0.84 for BLI-bright. The kappa values for non-experts were 0.86 for $\mathrm{LCl}, 0.97$ for $\mathrm{WL}$, and 0.78 for BLI-bright mode.

\section{Discussion}

$\mathrm{LCl}$ mode strengthens the color contrast between normal mucosa and colorectal lesions and it is brighter than other narrow-band light observations such as BLI and NBI. Thus, the color of the lesion becomes reddish and the surrounding mucosa becomes whitish. An earlier study reported an improvement in the visibility of diffuse redness of gastric mucosa in Helicobacter pylori induced gastritis with LCI [22]. Theoretically, reddish neoplastic lesions such as adenoma and cancer are detected in $\mathrm{LCI}$ mode according to the increase in reddish color in vascular rich areas of those lesions. On the other hand, whitish neoplastic lesions such as SSA/P are detected according to the increase in whitish color in vascular poor areas of those lesions. In addition, the residual liquid is not reddish compared to $\mathrm{BLI}$ and $\mathrm{NBI}$ modes. Generally, poor preparation is detected in $20-25 \%$ of all colonoscopies $[23,24]$. In those cases, NBI and BLI are not effective due to the reddish color of the residual liquid; however, $\mathrm{LCl}$ is thought to have increased efficacy in those cases. In our study, LCl showed higher polyp visibility scores than WL and BLI-bright in poor preparation cases.

Our previous study on polyp visibility between WL and BLIbright showed that, for non-experts, the polyp visibility scores 
Table 3 Mean polyp visibility scores for each clinical characteristic in WL, LCl and BLI-bright mode.

\begin{tabular}{|c|c|c|c|c|c|c|c|}
\hline & \multirow[b]{2}{*}{ Number of patients } & \multirow[b]{2}{*}{ WL } & \multirow[b]{2}{*}{$\mathrm{LCI}$} & \multirow[b]{2}{*}{ BLI } & \multicolumn{3}{|l|}{$P$ value } \\
\hline & & & & & WL vs LCI & WL vs BLI & LCI vs BLI \\
\hline Right-sided & 55 & $2.22 \pm 1.04$ & $2.61 \pm 1.07$ & $2.32 \pm 1.08$ & $<0.001$ & 0.12 & $<0.001$ \\
\hline Not right-sided & 46 & $2.91 \pm 1.17$ & $3.15 \pm 1.03$ & $3.32 \pm 0.99$ & $<0.001$ & $<0.001$ & 0.18 \\
\hline$\geq 10 \mathrm{~mm}$ & 38 & $2.65 \pm 1.15$ & $3.00 \pm 1.21$ & $2.82 \pm 1.13$ & $<0.001$ & 0.04 & 0.03 \\
\hline$<10 \mathrm{~mm}$ & 63 & $2.46 \pm 1.15$ & $2.67 \pm 1.13$ & $2.68 \pm 1.47$ & $<0.001$ & 0.07 & $<0.001$ \\
\hline Ad, Tis & 74 & $2.68 \pm 1.17$ & $3.06 \pm 1.04$ & $2.91 \pm 1.13$ & $<0.001$ & $<0.001$ & 0.009 \\
\hline SSA/P & 20 & $2.08 \pm 1.03$ & $2.20 \pm 0.99$ & $2.00 \pm 0.94$ & 0.04 & 0.43 & 0.02 \\
\hline Polypoid & 47 & $2.56 \pm 1.22$ & $2.81 \pm 1.15$ & $2.69 \pm 1.22$ & $<0.001$ & 0.02 & 0.06 \\
\hline Non-polypoid & 54 & $2.51 \pm 1.10$ & $2.90 \pm 1.03$ & $2.76 \pm 1.06$ & $<0.001$ & 0.03 & $<0.001$ \\
\hline Poor preparation & 23 & $2.42 \pm 1.13$ & $2.72 \pm 1.11$ & $2.31 \pm 1.09$ & 0.004 & 0.33 & $<0.001$ \\
\hline Good preparation & 78 & $2.55 \pm 1.19$ & $2.89 \pm 1.09$ & $2.79 \pm 1.18$ & $<0.001$ & 0.03 & $<0.001$ \\
\hline
\end{tabular}

WL: white light; LCI: linked color imaging; BLI: blue laser imaging; Ad: adenoma; Tis: intramucosal cancer; SSA/P: sessile serrated adenoma/polyp.
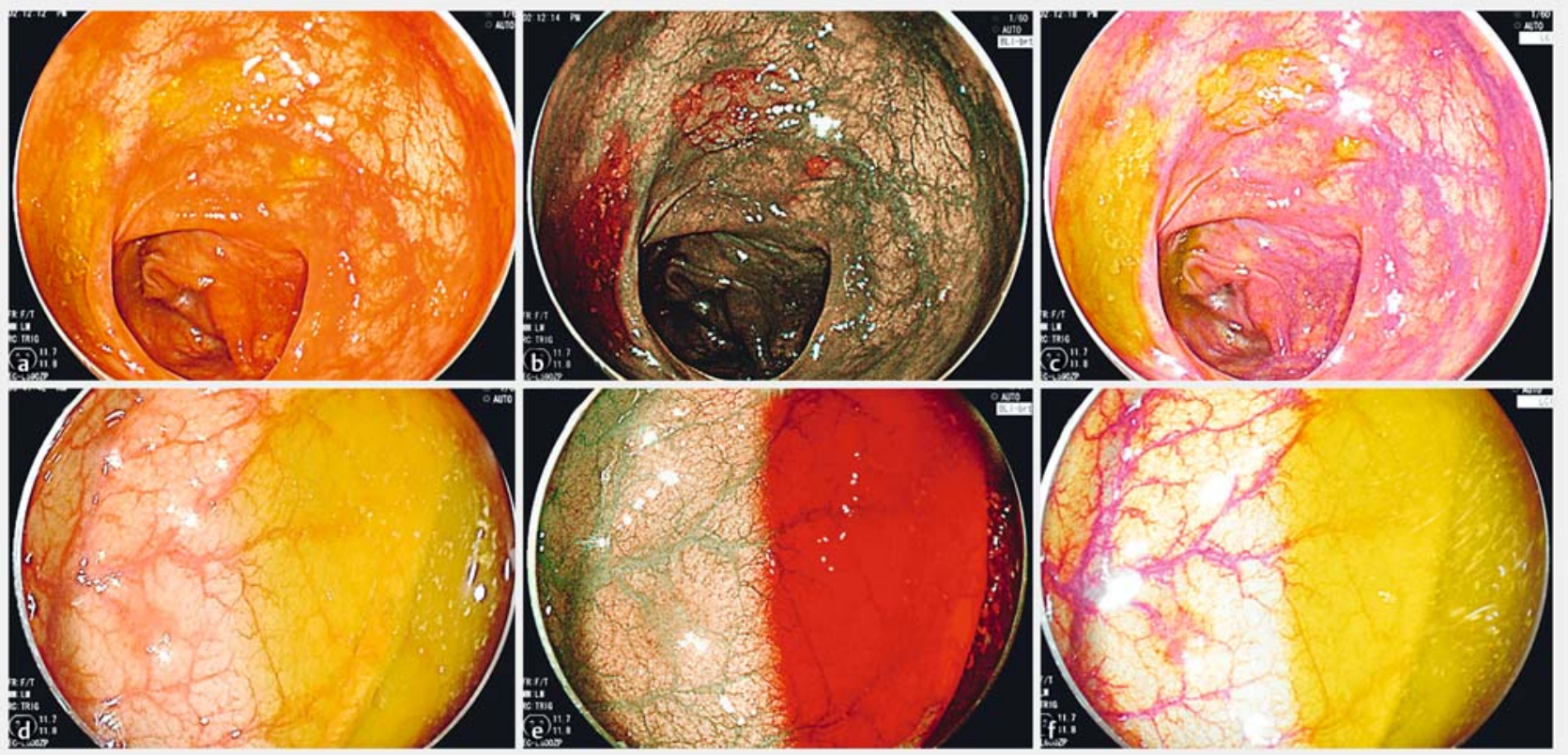

- Fig. 5 Case presentation. a A non-polypoid neoplastic polyp (SSA/P) in poor preparation, size $8 \mathrm{~mm}$ on the right-sided colon (ascending coIon). WL: score 2. b BLI-bright: score 2. Residual liquid became reddish and, when viewing the lesion from the oral side, the colon was dark. $\mathbf{c ~ L C I}$ score 3. Residual liquid was yellowish and, when viewing the lesion from the oral side, the colon was bright. $\mathbf{d}$ A non-polypoid neoplastic polyp (adenoma) in poor preparation, size $2 \mathrm{~mm}$ on the rectum. WL: score 2. e BLI-bright: score 3. $\mathrm{fLCl}$ : score 3.

for all clinical characteristics (location, size, histology, and morphology) in BLI-bright mode were significantly higher than those in WL. On the other hand, for experts, the scores for right-sided polyps, non-neoplastic polyps (including SSA/P), and polypoid polyps in BLI-bright mode were not higher than those in WL. One of the possible reasons for this was because the brightness of BLI-bright might not be sufficient for the wider right-sided colon. In the present study, the mean polyp visibility scores of $\mathrm{LCl}$ mode were higher than for WL for all clin- ical polyp parameters (location, size, histology, morphology). Compared to BLI-bright, those were higher in right-sided location and SSA/P histology, which were weak points of BLI-bright. Generally, right-sided polyps and non-polypoid polyps are sometimes difficult to be detected with WL, especially for nonexperts because of poor polyp visibility. However, LCl enables us to increase polyp visibility in polyps with various clinical characteristics and detect those polyps more easily than with WL for both experts and non-experts. 
Polyp detection is related to many factors such as lesion characteristics (location, morphology, size, and bowel preparation) and endoscopist's experience, especially insertion and withdrawal techniques, and polyp visibility. Among these factors, we believe that improvement in polyp visibility is one of the most important factors for improving polyp detection and the adenoma detection rate. In this study, only a small segment of video from the whole colonoscopic observation was used and we could control many factors including withdrawal speed and bowel preparation. We have previously reported video studies similar to this study $[16,25]$. One of our studies showed that the previous NBI system (EVIS LUCERA Spectrum system) did not increase polyp visibility and a more recent $\mathrm{NBI}$ system (EVIS LUCERA Elite system) increased polyp visibility [25]. Those findings are consistent with the failure of NBI studies on polyp detection [6,7]. We believe that this kind of video study is closer to a real colonoscopy than one using endoscopic images. In addition, the randomized video method is easier to be performed than a real polyp detection study. We believe that if this kind of video study is performed as a pre-study to evaluate the polyp visibility of a new modality, it is useful in deciding whether a subsequent polyp detection study should be performed. In this video study, LCl showed better polyp visibility than BLI-bright and WL. Thus, we believe that $\mathrm{LCl}$ is a good indication for a further polyp detection study.

There were some limitations to our study. Only patients receiving ESD in our center were enrolled in this study and all videos evaluated were recorded by the same expert endoscopist. Thus, there might be selection bias. This study was a singlecenter study and was performed by the review of videos to examine polyp visibility. Our final goal is to improve polyp detection, but the improvement in polyp visibility may not affect polyp detection in clinical cases because polyp detection is influenced by various factors other than polyp visibility.

In conclusion, our study showed that $\mathrm{LCl}$ improved polyp visibility compared to WL for both expert and non-expert endoscopists. It is useful for improving polyp visibility in any location and with any size, morphology, histology and preparation level.

\section{Acknowledgment}

We thank Dr. Norihiro Yamamoto, Dr. Mototsugu Kato, Dr. Takashi Hisabe, and Dr. Kenshi Yao for designing the study. We also thank Kubo Masahiro for assistance with procedures related to the Fujifilm LASEREO system. Moreover, we thank all members of the Department of Molecular Gastroenterology and Hepatology, Kyoto Prefectural University of Medicine for helping with this study.

\section{Competing interests}

Yoshito Itoh is affiliated with FUJIFILM Medical Co. Ltd. Yuji Naito received research grants from Otsuka Pharmaceutical Co., Ltd. and Takeda Pharmaceutical Co., Ltd. The other authors have no conflicts of interest to declare.
References

[1] Vogelstein B, Fearon ER, Hamilton SR et al. Genetic alterations during colorectal-tumor development. N Engl J Med 1988; 319: 525-532

[2] Saito Y, Uraoka T, Yamaguchi Y et al. A prospective, multicenter study of 1111 colorectal endoscopic submucosal dissections (with video). Gastrointest Endosc 2010; 72: 1217-1225

[3] Kudo S, Tamegai Y, Yamano H et al. Endoscopic mucosal resection of the colon: the Japanese technique. Gastrointest Endosc Clin N Am 2001; 11: 519-535

[4] Tanaka S, Haruma K, Oka S et al. Clinicopathological features and endoscopic treatment of superficially spreading colorectal neoplasms larger than 20 mm. Gastrointest Endosc 2001; 54: $62-66$

[5] van Rijn JC, Reitsma JB, Stoker J et al. Polyp miss rate determined by tandem colonoscopy: a systematic review. Am J Gastroenterol 2006; 101: $343-350$

[6] Adler A, Pohl H, Papanikolaou IS et al. A prospective randomised study on narrow-band imaging versus conventional colonoscopy for adenoma detection: does narrow-band imaging induce a learning effect? Gut 2008; 57: 59-64

[7] Rex DK, Helbig C. High yields of small and flat adenomas with highdefinition colonoscopes using either white light or narrow band imaging. Gastroenterology 2007; 133: 42-47

[8] Inoue T, Murano M, Murano N et al. Comparative study of conventional colonoscopy and pan-colonic narrow-band imaging system in the detection of neoplastic colonic polyps: a randomized controlled trial. J Gastroenterol 2008; 43: 45-50

[9] Horimatsu T, Sano Y, Tanaka S et al. Next-generation narrow band imaging system for colonic polyp detection: a prospective multicenter randomized trial. Int J Colorectal Dis 2015; 30: 947-954

[10] Chung S], Kim D, Song JH et al. Efficacy of computed virtual chromoendoscopy on colorectal cancer screening: a prospective, randomized, back-to-back trial of Fuji Intelligent Color Enhancement versus conventional colonoscopy to compare adenoma miss rates. Gastrointest Endosc 2010; 72: 136-142

[11] Pohl J, Lotterer E, Balzer C et al. Computed virtual chromoendoscopy versus standard colonoscopy with targeted indigocarmine chromoscopy: a randomised multicentre trial. Gastrointest Endosc 2009; 69: $734-741$

[12] Yoshida N, Hisabe T, Inada Y et al. The ability of a novel blue laser imaging system for the diagnosis of invasion depth of colorectal neoplasms. J Gastroenterol 2014; 49: $73-80$

[13] Yoshida N, Yagi N, Inada Y et al. The ability of a novel blue laser imaging system for the diagnosis of colorectal polyps. Dig Endosc 2014; 26: $250-258$

[14] Miyaki R, Yoshida S, Tanaka S et al. A computer system to be used with laser-based endoscopy for quantitative diagnosis of early gastric cancer. J Clin Gastroenterol 2015; 49: 108-115

[15] Osawa $\mathrm{H}$, Yamamoto $\mathrm{H}$. Present and future status of flexible spectral imaging color enhancement and blue laser imaging technology. Dig Endosc 2014; 26: (Suppl. 01): 105-115

[16] Yoshida N, Hisabe T, Hirose R et al. Improvement in the visibility of colorectal polyps by using blue laser imaging. Gastrointest Endosc 2015; 82: $542-549$

[17] Sun X, Dong T, Bi Y et al. Linked color imaging application for improving the endoscopic diagnosis accuracy: a pilot study. Sci Rep 2016; 6: 33473

[18] Yamashina T, Takeuchi Y, Uedo N et al. Diagnostic features of sessile serrated adenoma/polyps on magnifying narrow band imaging: a prospective study of diagnostic accuracy. J Gastroenterol Hepatol 2015; 30: $117-123$ 
[19] Participants in the Paris workshop. The Paris endoscopic classification of superficial neoplastic lesions: Esophagus, stomach, and colon-November 30 to December 1, 2002. Gastrointest Endosc 2003; 58: (Suppl) S3-S43

[20] Yoshida N, Naito Y, Murakami T et al. Safety and efficacy of a sameday low-volume $1 \mathrm{~L}$ PEG bowel preparation in colonoscopy for the elderly people and people with renal dysfunction. Dig Dis Sci 2016; 61: $3229-3235$

[21] Hamilton SR, Aaltonen LA, eds. World Health Organization classification of tumors. Pathology and genetics of tumours of the digestive system. Lyon, France: IARC Press; 2010: 104-109
[22] Dohi O, Yagi N, Onozawa Y et al. Linked color imaging improves endoscopic diagnosis of active Helicobacter pylori infection. Endosc Int Open 2016; 4: E800 - 805

[23] Froehlich F, Wietlisbach V, Gonvers JJ et al. Impact of colonic cleansing on quality and diagnostic yield of colonoscopy: the European Panel of Appropriateness of Gastrointestinal Endoscopy European multicenter study. Gastrointest Endosc 2005; 6: 378 - 384

[24] Harewood GC, Sharma VK, de Garmo P. Impact of colonoscopy preparation quality on detection of suspected colonic neoplasia. Gastrointest Endosc 2003; 58: 76 - 79

[25] Ogiso K, Yoshida N, Siah KTH et al. New generation narrow band imaging improves visibility of polyps: a colonoscopy video evaluation study. J Gastroenterol 2016; 51: 883-890 